\title{
Effect of haptoglobin on the treatment of chronic hepatitis B with interferon
}

\author{
LIN-LING LV ${ }^{1}$, YI-XUAN YANG ${ }^{2}$ and TONG-DONG SHI ${ }^{3}$ \\ ${ }^{1}$ Key Laboratory of Molecular Biology for Infectious Diseases (Ministry of Education); \\ ${ }^{2}$ Institute for Viral Hepatitis; ${ }^{3}$ Department of Infectious Diseases, The Second Affiliated Hospital, \\ Chongqing Medical University, Chongqing 400016, P.R. China
}

Received March 5, 2017; Accepted February 21, 2019

DOI: $10.3892 /$ etm.2019.7661

\begin{abstract}
There are two main types of drugs that are used to treat chronic hepatitis $\mathrm{B}(\mathrm{CHB})$, including interferon (IFN) and nucleotide analogues. IFN inhibits the virus through direct antiviral activity and via immune regulation, and it has been widely applied for the treatment of CHB and other infections. However, the efficiency of IFN therapy is not entirely satisfactory. The aim of the present study was to investigate the factors affecting IFN therapy. The plasma of patients with $\mathrm{CHB}$ treated with IFN was collected and divided into the virological response group and non-virological response group according to their virological response. Serum proteins of virologically responsive patients were compared before and after IFN therapy using isobaric tags for relative and absolute quantitation technology. ELISA was used to validate these results in the same sample. In in vitro cell experiments, HepG2.2.15 cells were transfected with haptoglobin (Hp)-targeting small interfering RNA to inhibit expression of the Hp protein, and reverse transcription-quantitative polymerase chain reaction and western blotting were utilized to detect hepatitis B virus (HBV)-DNA, IFN and downstream molecular changes in the cell supernatants. The Hp protein levels were demonstrated to be significantly lower following 48 weeks of IFN therapy, and the levels of $\mathrm{Hp}$ in patients in the virological response group were significantly lower than those in the non-virological response group. In in vitro cell experiments, following inhibition of Hp protein expression, significantly decreased levels of HBV-DNA, and elevated levels of IFN and its downstream molecules were observed. These findings suggest that $\mathrm{Hp}$ may be able to predict the efficacy of IFN therapy, and it may inhibit HBV clearance. There is an association between Hp
\end{abstract}

Correspondence to: Dr Tong-Dong Shi, Department of Infectious Diseases, The Second Affiliated Hospital, Chongqing Medical University, Building E, 1 Medical College Road, Yuzhong, Chongqing 400016, P.R. China

E-mail: shitongdong@aliyun.com

Key words: haptoglobin, chronic hepatitis B, interferon, blood, cell lines, small interfering RNA and IFN, which requires further clinical and laboratory studies to explore.

\section{Introduction}

Chronic hepatitis B (CHB) is the most common infectious chronic liver disease in humans, with a total of 240 million patients suffering from chronic hepatitis. $\mathrm{CHB}$ causes $>600,000$ mortalities each year from complications of end-stage liver disease and hepatocellular carcinoma (1). Antiviral treatments approved and available in China for $\mathrm{CHB}$ infection include standard or pegylated (Peg-) interferon (IFN) and nucleos(t) ide analogs (NAs), including an L-nucleoside analog (lamivudine), acyclic nucleoside phosphonates (adefovirdipivoxil and tenofovirdisoproxilfumarate) and a D-cyclopentane class nucleoside analog (entecavir). However, drug resistance and high cost of these drugs limits their long-term efficacy (2).

IFN, including Peg-IFN and unmodified IFN, has direct antiviral activity and immune stimulating properties. As a result, it has been widely used in recent decades. Studies have indicated that patients who received IFN therapy had a lower risk of decompensated liver diseases $(3,4)$. However, the available clinical data suggest that the antiviral efficacy of IFN therapy is currently not satisfactory. In clinical observation, the rate of effective IFN treatment was reported to be only $30-42 \%$ (5-7). Therefore, it is important to identify the factors that affect the treatment outcomes of patients with $\mathrm{CHB}$ who receive IFN therapy.

In the present study, isobaric tags for relative and absolute quantitation (iTRAQ)-based mass spectrometry (MS) was performed to quantify differentially expressed proteins (DEPs) between plasma samples from virological response (VR) patients before and after IFN therapy. DEPs are also sometimes referred to as 'aberrantly expressed proteins' in the present study when we wish to emphasize their biological and/or clinical relevance. The present iTRAQ analysis verified the likely importance of downregulated haptoglobin (Hp) expression after 48 weeks of IFN therapy. Serum Hp levels were lower in the VR group than in the non-VR group. Further in vitro experiments were conducted to verify the effects of Hp on IFN and on the clearance of hepatitis B virus (HBV). The present results suggest that $\mathrm{Hp}$ is a potentially useful target for the treatment of HBV and a marker for predicting the efficacy of IFN therapy. 


\section{Materials and methods}

Subjects and plasma collection. In the present study, 38 patients with CHB who received 48 weeks of Peg-IFN monotherapy $(180 \mu \mathrm{g} /$ week $)$ were followed up for $\geq 48$ weeks from September 2014-November 2015 at the Department of Infectious Diseases, The Second Affiliated Hospital, Chongqing, China. These patients were hepatitis B surface antigen (HBsAg) positive for $>6$ months and were currently HBsAg and/or DNA HBV positive at the time of treatment. Following 48 weeks of Peg-IFN treatment, for hepatitis B envelope antigen ( $\mathrm{HBeAg}$ )-positive patients with $\mathrm{CHB}$, virological response (VR) was defined as HBeAg seroconversion and an HBV DNA level $<2,000 \mathrm{IU} / \mathrm{ml}$. For HBeAg-negative patients with CHB, VR was defined as an HBV DNA level $<2,000 \mathrm{IU} / \mathrm{ml}$, in accordance with American Association for the Study of Liver Diseases guidelines (7). Following 48 weeks of IFN treatment, 10 VR patients and 28 non-VR patients were identified. The Ethics Committee of Chongqing Medical University (Chongqing, China) approved the present study. Written informed consent was obtained from all participants prior to treatment. Table I presents the patient demographics and clinical pathological data. Serum samples were collected, according to the guidelines set forth by the Human Proteome Organization Plasma Proteome Project (8), at the initiation, midpoint and end of therapy (weeks 0,24 and 48 , respectively), and stored at $-80^{\circ} \mathrm{C}$.

iTRAQ labeling. A total of 10 individual samples from VR patients taken at 0,24 and 48 weeks were mixed following treatment to create three sample pools. A ProteoPrep 20 Plasma Immunodepletion Kit (GE Healthcare Life Sciences, Shanghai, China) was used to deplete the most abundant proteins. A 2-D Quant kit (GE Healthcare Life Sciences) was used to measure the protein concentration of the immune depleted plasma. Protein samples were acetone precipitated at $-20^{\circ} \mathrm{C}$ for $1 \mathrm{~h}$ and dissolved in lysis buffer, denatured at $60^{\circ} \mathrm{C}$ for $1 \mathrm{~h}$ and blocked in $1 \mu \mathrm{l}$ cysteine at room temperature for $10 \mathrm{~min}$. Each sample was digested with trypsin solution at $37^{\circ} \mathrm{C}$ overnight, and then labeled with the iTRAQ tags with iTRAQ reagents (Applied Biosystems; Thermo Fisher Scientific, Inc., Waltham, MA, USA) as follows: week 0, $113 \mathrm{tag}$; week 24, $114 \mathrm{tag}$; and week 48,115 tag. The labeled samples were pooled prior to further analysis (9).

Peptide fractionation. The pooled iTRAQ labeled trypsin peptide samples were dissolved in $1 \%$ Pharmalyte and $8 \mathrm{M}$ urea and evenly coated on prehydrated immobilized $\mathrm{pH}$ gradient (IPG) strips (18 cm; pH 3-10). Samples were placed in an IPGphor system (GE Healthcare, Chicago, IL, USA) and subjected to isoelectric focusing at $68 \mathrm{kV} / \mathrm{h}$. The adhesive strip was then cut into 36 sections (thickness, $0.5 \mathrm{~cm}$ ). The peptides were extracted by incubating the gel sections in buffer A ( $2 \%$ acetonitrile, $0.1 \%$ formic acid) for $1 \mathrm{~h}$ at room temperature (10). These isolated peptides were freeze-dried, purified on a Discovery DSC-18 SPE column (Sigma-Aldrich; Merck KGaA, Darmstadt, Germany) and cryopreserved at $-80^{\circ} \mathrm{C}$ prior to further use.

$M S$. The purified peptides were dissolved in buffer A at room temperature for $20 \mathrm{~min}$ with vortex oscillation and loaded on to a C18-PepMap (Dionex; Thermo Fisher Scientific, Inc.) column (100 $\mu \mathrm{m} \times 3 \mathrm{~cm}, 3 \mu \mathrm{m}, 150 \mathrm{~A})$ of a Dionex Ultimate 3000 system. The peptides were eluted with the following concentration gradient: $4 \%$ buffer B $(98 \%$ acetonitrile, $0.1 \%$ formic acid) and $96 \%$ buffer A, $3 \mathrm{~min}$; $4-10 \%$ buffer B, $7 \mathrm{~min}$; 10-35\% buffer B, 55 min; 35-100\% buffer B, 25 min; $100 \%$ buffer B, $15 \mathrm{~min}$; $96 \%$ buffer A, $20 \mathrm{~min}$. The control flow rate was $0.3 \mu 1 / \mathrm{min}$ and the eluted fractions were automatically injected for MS analysis. The mass spectrometer was set in the positive ion mode at a mass range of $300-1,800 \mathrm{~m} / \mathrm{z}$. The switching time between MS scan and MS/MS event acquisition was set to $2 \mathrm{sec}$. The two most intensely charged peptides $>20$ counts were selected for MS/MS. Dynamic exclusion criteria was set to $30 \mathrm{sec}$.

Protein identification and quantification was performed using Protein Pilot v2.0 (AB SCIEX, Framingham, MA, USA). MS/MS data were processed by searching the International Protein Index Human database v3.77 (11). Methyl methanethiosulfonate was set as a fixed modification. Proteins with $\geq 2$ unique peptides were considered to be differentially expressed. The peak area of the iTRAQ tag molecule represents the relative quantification of the identified proteins in different samples, with a 95\% confidence interval and a P-value $<0.05$.

ELISA. Commercial ELISA kits were used to measure the serum levels of $\mathrm{Hp}$, hemoglobin subunit $\beta$ (HBB) and isoform 1 of fibrinogen $\alpha$ chain (FGA) according to the manufacturer's instructions. Human Hp ELISA kit (cat. no. ab108856), human HBB ELISA kit (cat. no. ab137972) and human FGA ELISA kit (cat. no. ab19079) were purchased from Abcam (Cambridge, UK).

Cell line. The HepG2.2.15 cell line, which was derived from the hepatoblastoma-derived cell line HepG2 and contains >1-unit length of the HBV genome integrated into the host genomes (12), was used in the present study. Cells were cultured at $5 \% \mathrm{CO}_{2}$ and $37^{\circ} \mathrm{C}$ in high-glucose Dulbecco's modified Eagle's medium (DMEM; GE Healthcare Life Sciences, Logan, UT USA) supplemented with $100 \mu \mathrm{g} / \mathrm{ml}$ streptomycin, $0.1 \%$ non-essential amino acids, $100 \mathrm{IU} / \mathrm{ml}$ penicillin, $1.0 \mathrm{mM}$ sodium pyruvate, $2 \mathrm{mM}$ glutamine and $10 \%$ fetal bovine serum (FBS; Gibco; Thermo Fisher Scientific, Inc.) (13).

Hp small interfering (si)RNA transfection. For functional studies, three siRNA duplexes against human Hp (SR302217A, 5'-GACCAGACCAAUGCAUAAGGCATT-3'; SR302217B, 5'-GGAGUGUACACACCUUAA ACAAUGAGA-3'; and SR302217C, 5'-CGGAUAUCGCAGAUGACGGCUGCC C-3') and a negative control (SR30004) were purchased from OriGene Technologies, Inc. (Rockville, MD, USA). Briefly, siRNA and Lipofectamine 2000 (Invitrogen; Thermo Fisher Scientific, Inc.) were diluted inmoderate Opti-MEM I medium (Gibco; Thermo Fisher Scientific, Inc.) for $5 \mathrm{~min}$ and incubated together for $20 \mathrm{~min}$ at room temperature. Subsequently $30-50 \%$ cells were plated into a 6-well plate and the transfection mixture added. The HepG2.2.15 cells were transfected with $20 \mathrm{nM}$ of Hp-specific siRNA and a negative control. Following $6 \mathrm{~h}$ of transfection, the medium was replaced with DMEM medium containing 10\% FBS and continued in culture for an additional $42 \mathrm{~h}$. Cells were harvested $48 \mathrm{~h}$ following 
Table I. Baseline characteristics and clinical data of patients receiving interferon therapy.

\begin{tabular}{|c|c|c|c|c|}
\hline \multirow[b]{2}{*}{ Characteristic } & \multicolumn{2}{|c|}{ Virological response $(n=10)$} & \multicolumn{2}{|c|}{ Non-virological response $(n=28)$} \\
\hline & 0 weeks & 48 weeks & 0 weeks & 48 weeks \\
\hline Age (years), mean \pm SD & $42.0 \pm 13.4$ & NA & $41.0 \pm 9.9$ & NA \\
\hline Sex (males) & $6(60.0 \%)$ & NA & $15(53.6 \%)$ & NA \\
\hline $\operatorname{HBeAg}(+)$ & $5(50.0 \%)$ & $0(0 \%)$ & $18(64.3 \%)$ & $13(46.4 \%)$ \\
\hline $\operatorname{ALT}(\mathrm{U} / \mathrm{l})$ & $105.8 \pm 37.8$ & $46.4 \pm 23.9$ & $132.8 \pm 60.0$ & $71.4 \pm 35.6$ \\
\hline HBV DNA $(\log I U / m l)$, mean \pm SD & $6.41 \pm 1.17$ & $2.68 \pm 0.51$ & $6.81 \pm 1.26$ & $4.69 \pm 0.80$ \\
\hline
\end{tabular}

SP, standard deviation; $\mathrm{HBeAg}$, hepatitis B envelope antigen; ALT, alanine amino transferase; HBV, hepatitis B virus.

transfection for western blot analysis and reverse transcription-quantitative polymerase chain reaction (RT-qPCR) analysis. All experiments were performed at least in triplicate.

Western blotting. Cells were lysed at $4^{\circ} \mathrm{C}$ with non-ionic detergent lysis buffer $(50 \mathrm{mM}$, pH 7.5 Tris-HCl; 0.5\% IGEPAL; $150 \mathrm{mM} \mathrm{NaCl} ; 1 \mathrm{mM}$, pH 8.0 EDTA; 0.5\% Triton X; $50 \mathrm{mM}$ sodium fluoride; $1 \mathrm{mM}$ sodium orthovanadate; and protease inhibitors). The lysates were centrifuged at $15,294 \mathrm{x} \mathrm{g}$ at $4^{\circ} \mathrm{C}$ for $30 \mathrm{~min}$. A 2-D Quant kit was used to determine the protein concentration. A total of $40 \mu \mathrm{g}$ protein/lane was separated by $10 \%$ SDS-PAGE and transferred to polyvinylidene difluoride membranes. The membranes were blocked at room temperature for $1 \mathrm{~h}$ with $5 \%$ non-fat milk in Tris-buffered saline solution with Tween-20 (TBS-T) and incubated overnight with the primary antibodies (all 1:500) in TBST plus 5\% bovine serum albumin (Beijing Solarbio Science \& Technology Co., Ltd., Beijing, China) at $4^{\circ} \mathrm{C}$. After washing three times with TBS-T buffer for $5 \mathrm{~min}$, the membranes were incubated with a horseradish peroxidase-conjugated goat anti-rabbit IgG as the secondary antibody $(1: 5,000)$ for $1 \mathrm{~h}$ at room temperature. Proteins were visualized with an enhanced chemiluminescencedetection system (Bio-Rad Laboratories, Hercules, CA, USA) $(9,14)$. The densitometry was analysed by ImageJ v1.51 (National Institutes of Health,Bethesda, MD, USA).Anti-Hp antibody(cat. no. ARG55106) was purchased from Arigo Biolaboratories (Taiwan, R.O.C. China), anti-GAPDH antibody (cat. no. ab181602) was purchased from Abcam, and the secondary antibody (cat. no. ATA0011) was purchased from ATgene Biotech (Chongqing, China).

$R T-q P C R$. Total RNA was isolated from the transfected cells using TRIzol reagent (Gibco; Thermo Fisher Scientific, Inc.) and reversed-transcribed using a Revert Aid First Strand cDNA Synthesis kit (Thermo Fisher Scientific, Inc.) according to the manufacturer's protocol. cDNA was quantified via qPCR with a KAPA SYBR FAST qPCR kit Master Mix (2X) Universal HBV detection kit (Roche Diagnostics, Basel, Switzerland) on a Roche LightCycler instrument (Roche Molecular Diagnostics, Pleasanton, CA, USA). The gene-specific primers used were as follows: Actin, Hp (cat. no. HP208336; forward, 5'-TGGCTAGTGGAGCAC TCGGTT-3', reverse, 5'-CAGGAAGTTTATCTCCAACAG CC-3'), MX dynamin like GTPase 1 (MX1; cat.no.HP206142; forward, 5'-GGCTGTTTACCAGACTCCGACA-3', reverse, 5'-CACA A A GCC TGGCAGCTCTCTA-3'), RNASEL (cat. no. HP214068; forward, 5'-AAGGCTGTTCAAGAA CTACACTTG-3', reverse, 5'-TGGATCTCCAGCCCA CTTGATG-3'), hepatic brain-expressed X (HBEX; cat. no. HP213124; forward, 5'-GCATAGGCTTGGAGAACC ACAG-3', reverse, 5'-CCGCAGACTATGACTCAACTG C-3'), ISG15 (cat. no. HP208303; forward, 5'-CTCTGAGCA TCCTGGTGAGGAA-3', reverse, 5'-AAGGTCAGCCAG AACAGGTCGT-3'), 2'-5'-oligoadenylate synthetase (OAS)2 (cat. no. HK209134; forward, 5'-GCTTCCGACAATCAA CAGCCAAG-3', reverse, 5'-CTTGACGATTTTGTGCCG CTCG-3'), eukaryotic translation initiation factor (EIF)-2 $\alpha$ (cat. no. HP215710; forward, 5'-CTGGACCTCATGCAG CTTTAGC-3', reverse, 5'-CTCCATAGTAGGAAGCTC CTGTC-3'), IFN $\beta 1$ (cat. no. HP205913; forward, 5'-CTT GGATTCCTACAAAGAAGCAGC-3', reverse, 5'-TCCTCC TTCTGGAACTGCTGCA-3'), OAS1 (cat. no. HP234311; forward, 5'-AGGAAAGGTGCTTCCGAG-3', reverse, 5'-GGACTGAGGAAGACAACCAGGT-3'), IFN $\alpha 1$ (cat. no. HP214678; forward, 5'-AGAAGGCTCCAGCCATCT CTGT-3', reverse, 5'-TGCTGGTAGAGTTCGGTGCAGA-3') and OAS3 (cat. no. HP209172; forward, 5'-CCTGATTCT GCTGGTGAAGCAC-3', reverse, 5'-TCCCAGGCAAAG ATGGTGAGGA-3'; OriGene Technologies, Inc.). Relative quantification of gene expression were calculated using the $2^{-\Delta \Delta \mathrm{Cq}}$ method (15).

Statistical analysis. Statistical analyses were performed using GraphPad Prism 6 (GraphPad Software, Inc., La Jolla, CA, USA) and SPSS v22 software (IBM Corp., Armonk, NY, USA). Continuous variables are presented as the mean \pm standard deviation. Differences between groups were analyzed using the Student's t-test and one-way ANOVA followed by Tukey's multiple comparison test. $\chi^{2}$ test or Fisher's exact test were used appropriately to compare categorical variables. $\mathrm{P}<0.05$ was considered to indicate a statistically significant difference.

\section{Results}

iTRAQ analysis of DEPs. In order to explore the differential expression profile of the plasma proteome in the treatment of patients with CHB with IFN therapy, iTRAQ-based MS 
Table II. Aberrantly expressed plasma proteins in virological response patients following 24 and 48 weeks of interferon therapy.

A, Downregulated proteins

\begin{tabular}{|c|c|c|c|c|c|}
\hline $\mathrm{N}$ & Name & 24 week/0 week 114/113 & P-value & 48 week/0 week 115/113 & P-value \\
\hline 1 & HP & 0.185 & $<0.001$ & 0.056 & $<0.001$ \\
\hline 2 & TMSL3 & 0.270 & 0.010 & 0.166 & 0.006 \\
\hline 3 & TLN1 & 0.149 & 0.021 & 0.191 & 0.053 \\
\hline 4 & APOA1 & 0.560 & $<0.001$ & 0.273 & $<0.001$ \\
\hline 5 & HPX & 0.550 & $<0.001$ & 0.291 & $<0.001$ \\
\hline 6 & HRG & 0.655 & 0.011 & 0.417 & $<0.001$ \\
\hline 7 & TPM4 & 0.275 & 0.041 & 0.425 & 0.062 \\
\hline 8 & IGHA2 & 0.421 & 0.021 & 0.437 & 0.032 \\
\hline 9 & $\mathrm{APOH}$ & 0.698 & 0.029 & 0.492 & 0.002 \\
\hline 10 & YWHAE & 0.334 & 0.044 & 0.511 & 0.034 \\
\hline 11 & GSN & 0.679 & 0.002 & 0.530 & $<0.001$ \\
\hline 12 & APOA4 & 0.570 & $<0.001$ & 0.575 & $<0.001$ \\
\hline
\end{tabular}

B, Upregulated proteins

\begin{tabular}{|c|c|c|c|c|c|}
\hline $\mathrm{N}$ & Name & 24 week/0 week 114/113 & P-value & 48 week/0 week $115 / 113$ & P-value \\
\hline 1 & HBB & 3.105 & $<0.001$ & 7.047 & $<0.001$ \\
\hline 2 & HBA2 & 2.938 & $<0.001$ & 5.916 & $<0.001$ \\
\hline 3 & FGA & 2.377 & $<0.001$ & 3.281 & $<0.001$ \\
\hline 4 & EEF2 & 1.959 & 0.002 & 2.512 & 0.001 \\
\hline 5 & FGG & 1.528 & 0.001 & 1.837 & $<0.001$ \\
\hline 6 & LGALS3BP & 2.032 & $<0.001$ & 1.75 & 0.030 \\
\hline
\end{tabular}

HP, haptoglobin; TLN1, talin-1; APOA1, apolipoprotein A-I; HPX, hemopexin; HRG, histidine-rich glycoprotein; IGHA2, immunoglobulin heavy chain variant (fragment); APOH, $\beta$-2-glycoprotein 1; YWHAE, isoform 1 of 14-3-3 protein $\varepsilon$; GSN, isoform 1 of gelsolin; APOA4, apolipoprotein A-IV; HBB, hemoglobin subunit $\beta$; HBA, hemoglobin subunit $\alpha$; FGA, isoform 1 of fibrinogen $\alpha$ chain; EEF2, elongation factor 2; FGG, isoform $\gamma$-B of fibrinogen $\gamma$ chain; LGALS3BP, cDNA FLJ53478, highly similar to Galectin-3-binding protein.

was utilized to analyze serum proteins at 0,24 and 48 weeks from VR patients receiving IFN therapy. DEPs were defined using a $1.3(1 \times 1.3)$ and $0.77(1 / 1.3)$ cut-off in accordance with commonly adopted iTRAQ-based MS conventions. This value has been employed in other large-scale protein identification and quantification studies using the iTRAQ approach $(9,10,14,16,17)$. Briefly, proteins with iTRAQ ratios $<0.77$ were considered to be underexpressed, where as those $>1.3$ were considered overexpressed (10). A total of 522 non-extension proteins were identified. Based on the above values, 18 proteins were revealed to be differentially expressed between the groups, with 12 proteins being downregulated and 6 proteins being upregulated. Information on these proteins is presented in Table II.

Validation of DEPs. Hp, HBB and FGA were selected from the DEPs for further validation. ELISA assays were employed to determine the plasma levels of HP, HBB and FGA from the same sample set utilized for the iTRAQ analysis. The ELISA results were consistent with the results of iTRAQ, as the plasma levels of $\mathrm{Hp}$ were significantly decreased and HBB and FGA were increased following IFN therapy (Fig. 1A-C).
$H p$ reflects the efficacy of IFN in the treatment of $C H B$. The analysis indicated that Hp exhibited the greatest reduction among the DEPs. ELISA was utilized to detect the plasma levels of $\mathrm{Hp}$ in VR patients and non-VR patients following 48 weeks of treatment. The plasma levels of Hp in VR patients were significantly lower than in non-VR patients (Fig. 1D). Hp reflects the efficacy of IFN in the treatment of $\mathrm{CHB}$.

Validation of interference effect. In order to explore the effect of the Hp protein on the hepatitis B virus, Hp-specific siRNA was used to inhibit the expression of the Hp gene in HepG2.215 cells. RT-qPCR and western blotting were utilized to verify the effect of siRNA knockdown at the gene and protein level. As presented in Fig. 2, the expression of Hp was significantly reduced at the gene and protein levels in the siRNA knockdown groups, compared with the control group.

Association of $\mathrm{Hp}$ with $\mathrm{HBV}$ replication. In order to explore whether $\mathrm{Hp}$ has an effect on the replication of HBV, the expression of $\mathrm{Hp}$ was inhibited with $\mathrm{Hp}$-specific siRNA to observe changes in the levels of HBV DNA in HepG2.2.15 cell supernatants. Following $48 \mathrm{~h}$ of incubation, the $\mathrm{Hp}$ 
A

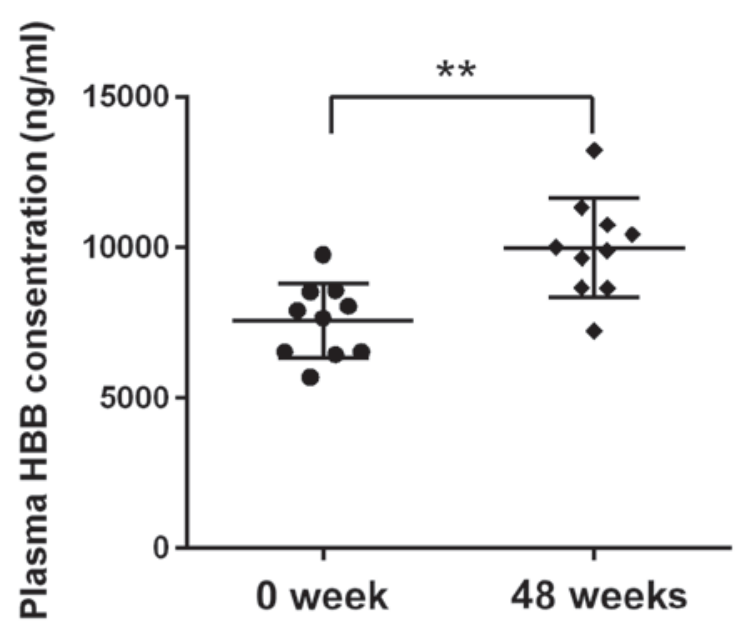

C

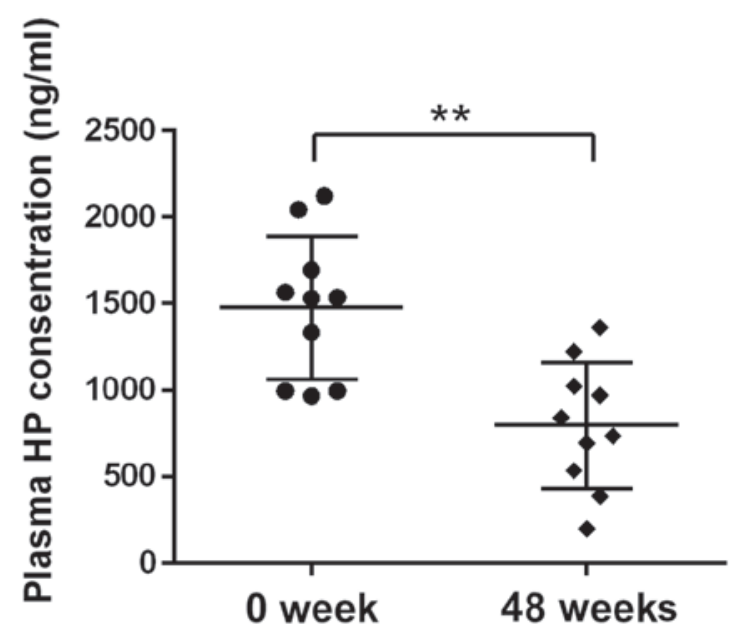

B

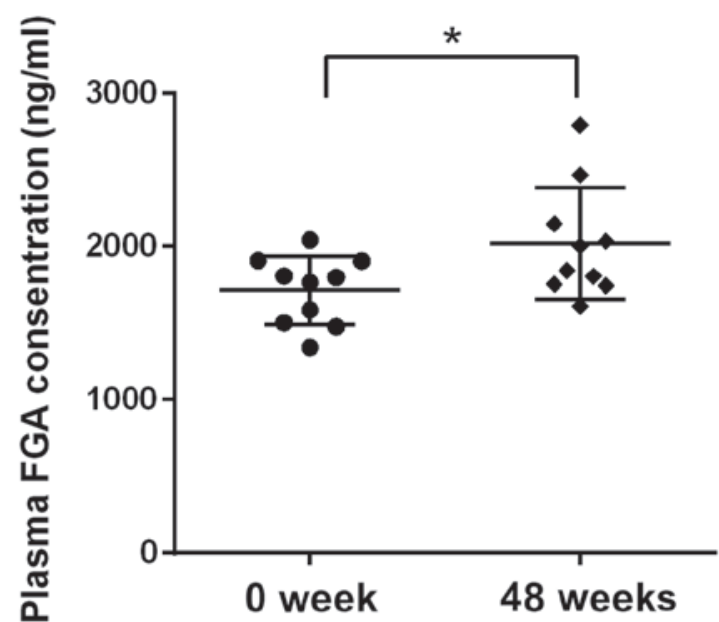

Figure 1. ELISA verification of HP, HBB and FGA. (A-C) ELISA verification of iTRAQ results. Samples tested were the same as those used in the iTRAQ experiments. (D) ELISA verification of HP in VR and non-VR patients. The plasma levels of HP in VR patients were significantly lower than in non-VR patients. Data are presented as the mean \pm standard deviation. ${ }^{*} \mathrm{P}<0.05,{ }^{* *} \mathrm{P}<0.01$. HP, haptoglobin; HBB, hemoglobin subunit $\beta$; FGA, isoform 1 of fibrinogen $\alpha$ chain; iTRAQ, isobaric tags for relative and absolute quantitation; VR, virological response.

siRNA-transfected HepG2.2.15 cells exhibited reduced HBV DNA levels (Fig. 3).

Hp-mediated alteration of IFN and IFN-Induced downstream signaling pathways. In the treatment of hepatitis B with IFN, the level of $\mathrm{Hp}$ in the different response groups was varied. It was demonstrated that HBV levels decreased significantly when the expression of Hp was downregulated, and it was hypothesized that $\mathrm{Hp}$ may affect the production of IFN. Hp-specific siRNA was used to knockdown Hp expression and, following $48 \mathrm{~h}$ of culture, extracted mRNA from the cells was used to detect the levels of IFN RNA. The results indicated that IFN $\beta 1$ levels were significantly increased in comparison with the control group (Fig. 4). The expression levels of downstream IFN-stimulated genes in response to Hp inhibition were also detected and, as presented in Fig. 4, knockdown of Hp expression induced increased mRNA levels of five downstream IFN-stimulated genes (OAS1, OAS3, Mx1, ribonuclease Land HBEX). However, four other effectors (IFN $\alpha 1$, ISG15, EIF-2 $\alpha$ and OAS2) exhibited only minimally altered mRNA levels in response to HP downregulation.

\section{Discussion}

Peg-IFN and nucleoside (acid) analogues are currently the two major antiviral drugs utilized for the treatment of hepatitis B. Although NAs are well tolerated, long treatment periods are required to halt the risk of relapse. Peg-IFN provides a greater likelihood of improved outcomes in a limited 48-week period and with a sustained viral response, but its tolerance is poor and Peg-IFN only exhibits efficacy for a number patients (18). Many factors may affect the Peg-IFN effect, such as baseline HBV DNA level, HBV genotype, alanine amino transferase (ALT) level, sex, age and patient genetic factor (19); however, the exact molecular mechanism is not well known. The efficiency of IFN therapy in the treatment of hepatitis B following 48 weeks is only $30-42 \%$ (77), and requires further investigation. HBV genotype, and pre-treatment HBV DNA and ALT levels have been developed to help select those patients best suited for Peg-IFN treatment $(18,20)$.

An aim of the present study was to elucidate the factors that affect the efficacy of IFN therapy. A previous epidemiological study has indicated that the results of antiviral 
A
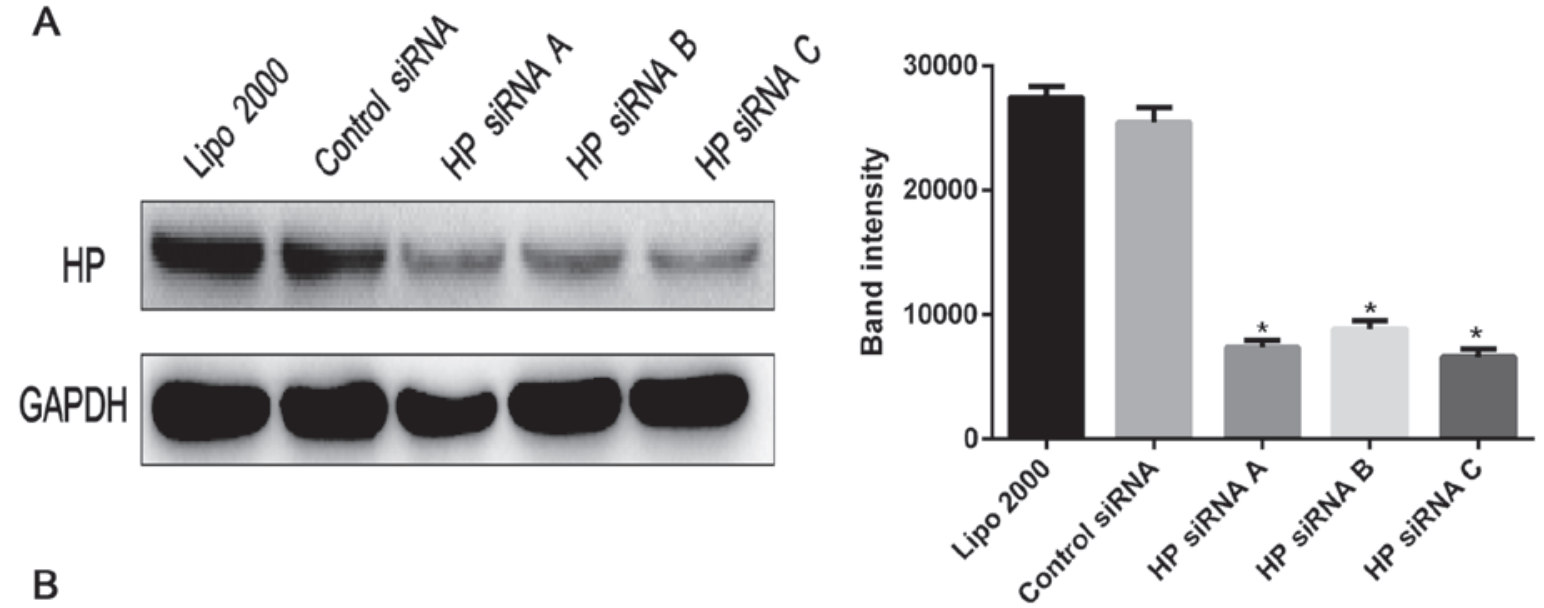

B

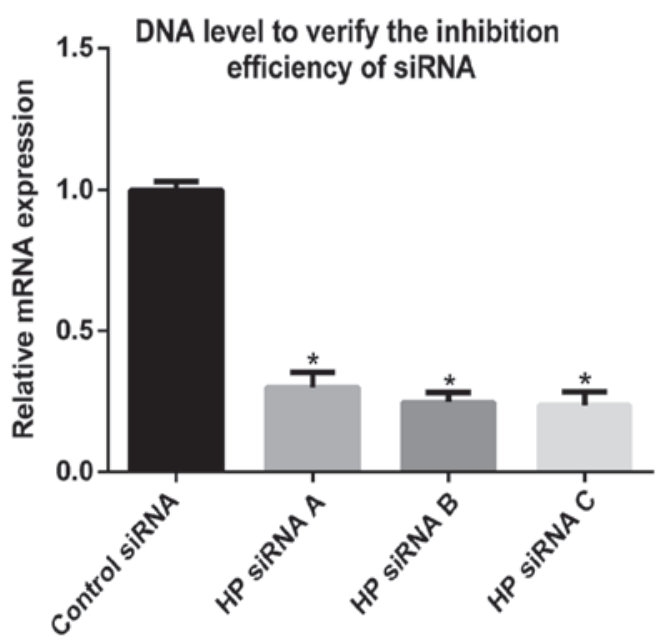

Figure 2. Evaluation of inhibition efficiency. HepG2.215 cells were transfected with HP-targeting siRNAs. (A) Western blot analysis of intracellular HP and its respective gray scale analysis results. (B) Reverse transcription-quantitative polymerase chain reaction analysis of intracellular HP. Representative images are presented. Data are presented as the mean \pm standard deviation. ${ }^{*} \mathrm{P}<0.05$ vs. control siRNA. HP, haptoglobin; siRNA, small interfering RNA.

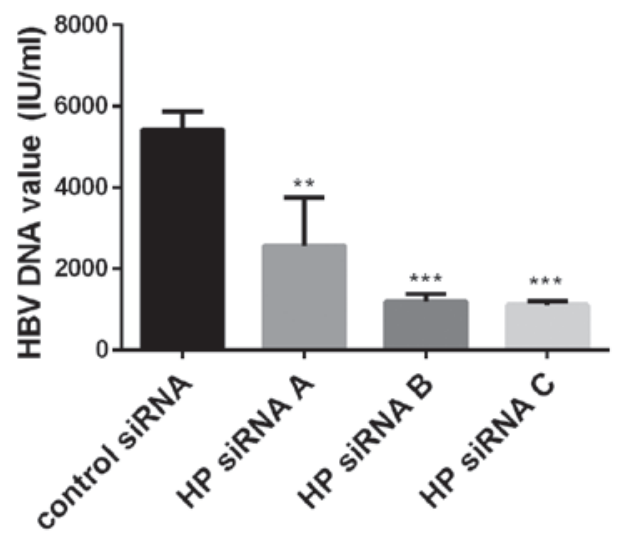

Figure 3. Functional evaluation of the role of HP in HBV replication in HepG2.215 cells. The supernatant HBV DNA level in HP-knockdown cells is significantly decreased, compared with control siRNA. Data are presented as the mean \pm standard deviation. ${ }^{* *} \mathrm{P}<0.01,{ }^{* * *} \mathrm{P}<0.001$ vs. control siRNA. $\mathrm{HP}$, haptoglobin; HBV, hepatitis B virus; siRNA, small interfering RNA.

therapy are affected by many factors, such as viral and host factors (20). In order to explore these differences, many novel labeling methods such as iTRAQ have been recommended by the proteomics community to provide deeper proteome coverage and convenient biomarker discovery $(21,22)$. In the present study, patients with CHB were treated with IFN. According to the effects of IFN treatment, patients were divided into the VR and non-VR groups. iTRAQ was utilized in order to screen the differential expression of proteins in the VR patients at 0,24 and 48 weeks of IFN therapy.

$\mathrm{Hp}$ is a 2-glycoprotein acute phase reactant which binds to free hemoglobin and forms a stoichiometrically stable complex (23). Hpis expressed in many tissues and cell types, but the major source is the liver (24). Viagel electrophoresis, Hpis divided into three main types: Hp 1-1, Hp 2-1, and Hp 2-2 (25). The major biological function of $\mathrm{Hp}$ is to prevent hemoglobin-mediated renal parenchymal injury and the loss of iron following intravascular hemolysis by binding to hemoglobin with very high affinity in an equimolar ratio (26). Other functions include antioxidant activity, prevention of renal damage, antibacterial activity, inhibition of nitric oxide, inhibition of prostaglandins and angiogenesis (27).

The present analysis indicated that Hp was the most markedly reduced DEP. Following ELISA verification, it was also identified that $\mathrm{Hp}$ decreased significantly following treatment. Meanwhile, in patients with good response, the level of $\mathrm{Hp}$ also decreased significantly. This suggested that the level of Hp may reflect the efficacy of IFN treatment of CHB. 


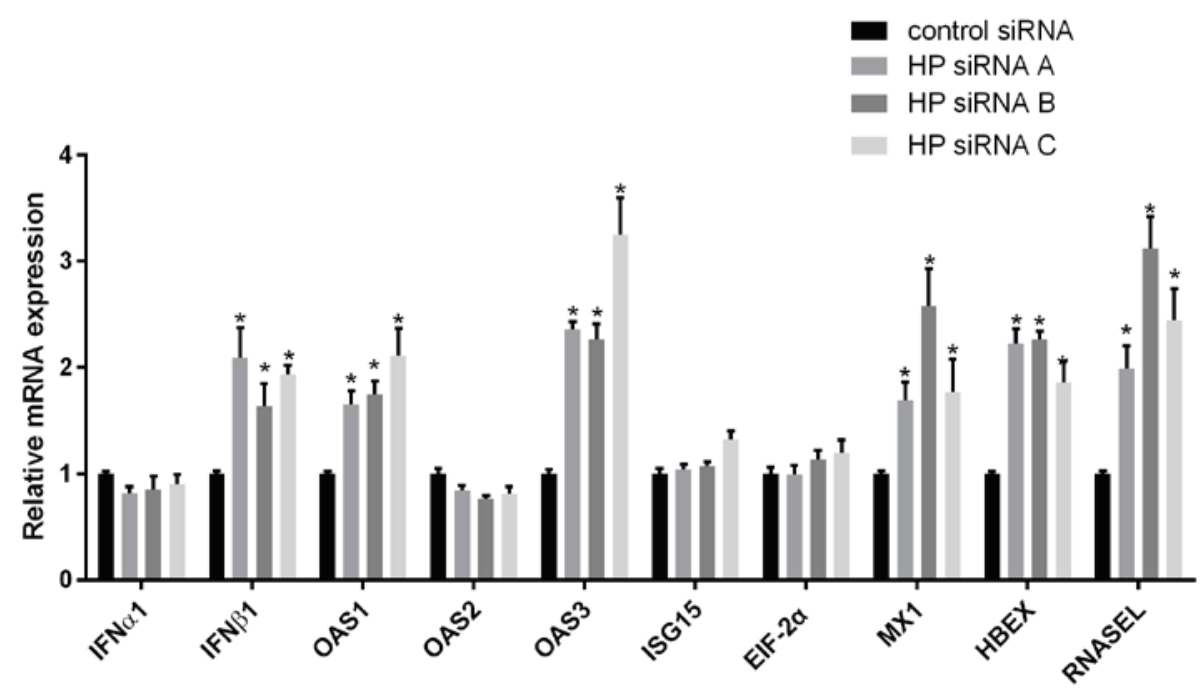

Figure 4. Reverse transcription-quantitative polymerase chain reaction was used to detect the relative mRNA expression levels of IFN and downstream IFN-stimulated genes following knockdown of HP. The relative mRNA expression levels of type I IFN and five downstream IFN-stimulated genes were increased, and no significant changes were observed for the other IFN-stimulated genes. Data are presented as the mean \pm standard deviation. ${ }^{*} \mathrm{P}<0.05$ vs. control siRNA. IFN, interferon; HP, haptoglobin; siRNA, small interfering RNA; OAS, 2'-5'-oligoadenylate synthetase; EIF, eukaryotic translation initiation factor; MX1, MX dynamin like GTPase 1; HBEX, hepatic brain-expressed X; RNASEL, ribonuclease L.

A number of previous studies have demonstrated that inflammation, trauma, burns and tumors are often accompanied by an increase in plasma levels of $\mathrm{Hp}$ binding to the plasma protein $(23,28)$. Therefore, Hp has antioxidant and anti-hemolytic function. Reduced levels of Hp maybe associated with hemolysis or liver injury (29). This is in accordance with the present research results. In the present study, it was demonstrated that ALT was lower in the VR group than the NON-VR group. If a significant difference had been observed in a comparison between the experimental group and another normal patient group, it would have been more convincing. The present study indicates that $\mathrm{Hp}$ was decreased significantly in the group with a good response to IFN therapy, which exhibited a decreased level of HBV-DNA. This suggests that Hp may have an inhibitory effect on viral clearance. It has been demonstrated that the Hp level in patients with hepatitis is different from that in normal subjects. In hepatitis $\mathrm{C}$ virus infection, increased levels of Hp1-1 have been reported (30). Patients with higher levels of Hp2-2 produce lower levels of hepatitis B antibody (30). In vitro cell experiments further verified that, in HepG2.215 cells, the amount of HBV-DNA in the supernatant was detected following inhibited expression of $\mathrm{Hp}$. In cell experiments, where a lower expression of Hp was present, the amount of virus was demonstrated to decrease, indicating that $\mathrm{Hp}$ inhibited viral clearance.

It has previously been demonstrated that Hp functions as an immune system modulator (27). Hp establishes Th1-Th2 balance by enhancing the Th1 cellular response in vitro (31). Via the cluster of differentiation (CD)11b/CD18 (MAC 1) receptor, $\mathrm{Hp}$ binds to granulocytes, monocytes, T CD8 cells and natural killer cells, and can regulate the function of MAC 1 dependent cells (32). The Hp-hemoglobin complex is migrated by binding to the CD163 receptors on the surface of monocytes and macrophages $(33,34)$. In the present study, the plasma levels of HP following IFN treatment were significantly lower than prior to treatment. It was hypothesized that the level of Hp may have some association with IFN.
In vitro experiments demonstrated that the levels of IFN in HepG2.2.15 cells increased when Hp expression was inhibited. It was speculated that the presence of Hp may inhibit IFN, but it is not clear how specific pathways may affect it. The effects of Hp levels on the levels of downstream molecules of IFN were also explored. With the siRNA-meditated under expression of Hp, levels of IFN $\beta$ increased and the downstream molecules of IFN increased significantly. This data demonstrated that the presence of Hp not only inhibits the production of IFN, but also inhibits the production of downstream signaling molecules.

The present findings suggest the following: i) Hp was the most downregulated differential protein following IFN treatment, the level of $\mathrm{Hp}$ is decreased in patients with low HBV levels and the level of Hp may reflect the therapeutic effect of IFN. ii) Patients with low HBV levels exhibit a decreased level of $\mathrm{Hp}$; in the in vitro experiments, the level of HBV-DNA was significantly decreased when Hp expression was inhibited, suggesting that Hp may promote the replication of HBV and inhibit the clearance of HBV. iii) Hp exhibited the greatest downregulation following IFN treatment, and affects the production of IFN and its downstream molecules; suggesting that there is an association between $\mathrm{Hp}$ and IFN. In future in vitro experiments, we may alter exogenous IFN levels to explore whether this inhibits the clearance of HBV.

The present study also had a number of limitations. Firstly, changes in serum protein levels following treatment with other drugs (nucleotide analogues) were not excluded. Secondly, there was a lack of normal patient control samples. A more rigorous experimental design is required in future experiments, as the present conclusions are valuable, and merit further exploration.

\section{Acknowledgements}

Not applicable. 


\section{Funding}

The present study was supported by National Nature Science Foundation of China (grant no. 81172804).

\section{Availability of data and materials}

The datasets used and/or analyzed during the present study are available from the corresponding author on reasonable request.

\section{Authors' contributions}

LL made substantial contributions to the design of the present study and to the acquisition, analysis and interpretation of the data. LL, TS and YY participated in the design of the study and in the acquisition, analysis and interpretation of data. All authors have read and approved the final manuscript.

\section{Ethics approval and consent to participate}

The Ethics Committee of Chongqing Medical University (Chongqing, China) approved this study. Written informed consent was obtained from all participants prior to treatment.

\section{Patient consent for publication}

Not applicable.

\section{Competing interests}

The authors declare that they have no competing interests.

\section{References}

1. GBD 2013 Mortality and Causes of Death Collaborators: Global, regional, and national age-sex specific all-cause and cause-specific mortality for 240 causes of death, 1990-2013: A systematic analysis for the global burden of disease study 2013 Lancet 385: 117-171, 2015.

2. Torresi J and Locarnini SA: New therapeutic strategies in the treatment of hepatitis B virus infection. Expert Opin Investig Drugs 8: 289-305, 1999.

3. van Zonneveld M, Honkoop P, Hansen BE, Niesters HG, Darwish Murad S, de Man RA, Schalm SW and Janssen HL: Long-term follow-up of alpha-interferon treatment of patients with chronic hepatitis B. Hepatology 39: 804-810, 2004.

4. Sung JJ, Tsoi KK, Wong VW, Li KC and Chan HL: Meta-analysis: Treatment of hepatitis B infection reduces risk of hepatocellular carcinoma. Aliment Pharmacol Ther 28: 1067-1077, 2008.

5. Lau GK, Piratvisuth T, Luo KX, Marcellin P, Thongsawat S, Cooksley G, Gane E, Fried MW, Chow WC, Paik SW, et al: Peginterferon Alfa-2a, lamivudine, and the combination for $\mathrm{HBeAg}$-positive chronic hepatitis B. N Engl J Med 352: 2682-2695, 2005.

6. Janssen HL, van Zonneveld M, Senturk H, Zeuzem S, Akarca US, Cakaloglu Y, Simon C, So TM, Gerken G, de Man RA, et al: Pegylated interferon alfa-2b alone or in combination with lamivudine for $\mathrm{HBeAg}$-positive chronic hepatitis B: A randomised trial. Lancet 365: 123-129, 2005.

7. Terrault NA, Bzowej NH, Chang KM, Hwang JP, Jonas MM, Murad MH and American Association for the Study of Liver Diseases: AASLD guidelines for treatment of chronic hepatitis B. Hepatology 63: 261-283, 2016.

8. Rai AJ, Gelfand CA, Haywood BC, Warunek DJ, Yi J, Schuchard MD, Mehigh RJ, Cockrill SL, Scott GB, Tammen H, et al: HUPO plasma proteome project specimen collection and handling: Towards the standardization of parameters for plasma proteome samples. Proteomics 5: 3262-3277, 2005.
9. Zhang $\mathrm{H}$, Li H, Yang Y, Li S, Ren H, Zhang D and Hu H: Differential regulation of host genes including hepatic fatty acid synthase in HBV-transgenic mice. J Proteome Res 12: 2967-2979, 2013.

10. Yang Y, Toy W, Choong LY, Hou P, Ashktorab H, Smoot DT, Yeoh KG and Lim YP: Discovery of SLC3A2 cell membrane protein as a potential gastric cancer biomarker: Implications in molecular imaging. J Proteome Res 11: 5736-5747, 2012.

11. Kersey PJ, Duarte J, Williams A, Karavidopoulou Y, Birney E and Apweiler R: The international protein index: An integrated database for proteomics experiments. Proteomics 4: 1985-1988, 2004.

12. Sells MA, Chen ML and Acs G: Production of hepatitis B virus particles in Hep G2 cells transfected with cloned hepatitis B virus DNA. Proc Natl Acad Sci USA 84: 1005-1009, 1987.

13. Lepiller Q, Abbas W, Kumar A, Tripathy MK and Herbein G: HCMV activates the IL-6-JAK-STAT3 axis in HepG2 cells and primary human hepatocytes. PLoS One 8: e59591, 2013.

14. She S, Xiang Y, Yang M, Ding X, Liu X, Ma L, Liu Q, Liu B, Lu Z, $\mathrm{Li} \mathrm{S}$, et al: C-reactive protein is a biomarker of AFP-negative HBV-related hepatocellular carcinoma. Int J Oncol 47: 543-554, 2015.

15. Schmittgen TD and Livak KJ: Analyzing real-time PCR data by the comparative C(T) method. Nat Protoc 3: 1101-1108, 2008.

16. Zhou C, Simpson KL, Lancashire LJ, Walker MJ, Dawson MJ, Unwin RD, Rembielak A, Price P, West C, Dive C and Whetton AD: Statistical considerations of optimal study design for human plasma proteomics and biomarker discovery. J Proteome Res 11: 2103-2113, 2012.

17. Chong PK, Lee H, Zhou J, Liu SC, Loh MC, So JB, Lim KH, Yeoh KG and Lim YP: Reduced plasma APOA1 level is associated with gastric tumor growth in MKN45 mouse xenograft model. J Proteomics 73: 1632-1640, 2010.

18. Osiowy C, Coffin C and Andonov A: Review of laboratory tests used in monitoring hepatitis B response to pegylated interferon and nucleos(t)ide analog therapy. Curr Treat Options Infect Dis 8: 177-193, 2016.

19. Buster EH, Hansen BE, Lau GK, Piratvisuth T, Zeuzem S, Steyerberg EW and Janssen HL: Factors that predict response of patients with hepatitis B e antigen-positive chronic hepatitis B to peginterferon-alfa. Gastroenterology 137: 2002-2009, 2009.

20. Gygi SP, Rist B, Gerber SA, Turecek F, Gelb MH and Aebersold R: Quantitative analysis of complex protein mixtures using isotope-coded affinity tags. Nat Biotechnol 17: 994-999, 1999.

21. Ross PL, Huang YN, Marchese JN, Williamson B, Parker K, Hattan S, Khainovski N, Pillai S, Dey S, Daniels S, et al: Multiplexed protein quantitation in Saccharomyces cerevisiae using amine-reactive isobaric tagging reagents. Mol Cell Proteomics 3: 1154-1169, 2004.

22. Javid J: Human haptoglobins. Curr Top Hematol 1: 151-192, 1978.

23. Andersen CBF, Stødkilde K, Sæderup KL, Kuhlee A, Raunser S, Graversen JH and Moestrup SK: Haptoglobin. Antioxid Redox Signal 26: 814-831, 2017.

24. Smithies O: Zone electrophoresis in starch gels: Group variations in the serum proteins of normal human adults. Biochem J 61: 629-641, 1955.

25. Lim SK: Consequences of haemolysis without haptoglobin. Redox Rep 6: 375-378, 2001.

26. Sadrzadeh SM and Bozorgmehr J: Haptoglobin phenotypes in health and disorders. Am J Clin Pathol 121 (Suppl): S97-S104, 2004.

27. Wang F, Huang W and Li A: Serum haptoglobin suppresses T-lymphocyte functions following burns. Chin Med Sci J 11: 180-183, 1996.

28. Peta V, Tse C, Perazzo H, Munteanu M, Ngo Y, Ngo A, Ramanujam N, Verglas L, Mallet M, Ratziu V, et al: Serum apolipoprotein A1 and haptoglobin, in patients with suspected drug-induced liver injury (DILI) as biomarkers of recovery. PLoS One 12: e0189436, 2017.

29. Louagie H, Delanghe J, Desombere I, De Buyzere M, Hauser P and Leroux-Roels G: Haptoglobin polymorphism and the immune response after hepatitis B vaccination. Vaccine 11: 1188-1190, 1993.

30. Arredouani M, Matthijs P, Van Hoeyveld E, Kasran A, Baumann H, Ceuppens JL and Stevens E: Haptoglobin directly affects $\mathrm{T}$ cells and suppresses $\mathrm{T}$ helper cell type 2 cytokine release. Immunology 108: 144-151, 2003.

31. El Ghmati SM, Van Hoeyveld EM, Van Strijp JG, Ceuppens JL and Stevens EA: Identification of haptoglobin as an alternative ligand for CD11b/CD18. J Immunol 156: 2542-2552, 1996. 
32. Goldstein JI, Goldstein KA, Wardwell K, Fahrner SL, Goonan KE, Cheney MD, Yeager MP and Guyre PM: Increase in plasma and surface CD163 levels in patients undergoing coronary artery bypass graft surgery. Atherosclerosis 170: 325-332, 2003.
33. Kristiansen M, Graversen JH, Jacobsen C, Sonne O, Hoffman HJ, Law SK and Moestrup SK: Identification of the haemoglobin scavenger receptor. Nature 409: 198-201, 2001 